\title{
Can a Virtual Agent provide good Emotional Support?
}

\author{
Exploring whether personality or identity effect the perceived supportiveness of a message \\ Kirsten A. Smith \\ University of Southampton \\ Southampton, UK \\ kirsten.smith@soton.ac.uk \\ Judith Masthoff \\ University of Aberdeen \\ Aberdeen, UK \\ j.masthoff@abdn.ac.uk
}

\begin{abstract}
In this study we explore whether an emotional support message sent to an informal carer by a Virtual Agent provides good quality emotional support, compared to the same message sent by a friend or sister with whom they have either a close, medium, or distant relationship. We also explore whether these judgements are affected by personality. Participants recruited from Mechanical Turk rated an emotional support message for Suitability, provided qualitative feedback about their rating and then completed a personality measure. We found that the support message was rated worst when it came from the Computer, Distant-sister and Close-friend. While these were rated worse, they were not rated poorly, implying that support from a computer is valuable. There were three effects for personality which did not vary with the support giver's Identity: agreeableness and emotional stability had a positive correlation with 3 sub-scales of supportiveness. A thematic analysis of comments revealed that people prefer emotional support from a human; they like empathy; support from close friends means more; they prefer personalised support; and they have higher expectations from family over friends.
\end{abstract}

$\mathrm{HCl}$, Virtual agents, carers, affective computing, emotional support, personality, eHealth.

\section{INTRODUCTION}

Digital health applications are among the most timely and helpful innovations of the age that have potential to provide a comprehensive approach to integrated care (World Health Organization and others 2016) - yet there is the danger of them being impersonal. The repercussions of removing the face-toface interactions between two people is not well understood. In this study we explore whether a text-based emotional support message is perceived differently depending on who sent it, and whether this varies with the personality of the reader. The results of this study can be used to inform the future development of emotional support systems for people experiencing stress.

It is possible to insert empathetic content into an eHealth application using a virtual agent (VA). VAs have potential to improve engagement with $e-$ Health interventions by expressing empathy and providing affective responses to user input (Scholten et al. 2017). Good quality emotional support has been found to reduce negative affect (Meyer and Turner 2002), though inappropriate support could harm the relationship between the support giver and receiver (Burleson and Kunkel 1996). This is particularly critical for VAs as it might break the user's suspension of disbelief (that a computer actually empathises with the user) (Scholten et al. 2017) and impact upon the user's willingness to engage in further activities.
This type of emotional support may be particularly useful in the carer domain. It has been widely found that carers exhibit more severe physical and mental health problems than non-carers of a similar demographic (e.g. Savage and Bailey 2004, Schoenmakers et al. 2010, Caqueo-Urízar et al. 2009, Vitaliano et al. 2003). This is exacerbated by the fact that carers are often elderly themselves and/or suffer from medical conditions themselves. In the UK, 1 in 5 people aged 50-64 are carers; 1 in 4 women and 1 in 6 men. $63 \%$ of carers have a long-term health condition (compared to $51 \%$ of non-carers) (Carers UK 2015).

In supporting carers it is thus important to recognise that they are not a homogenous group carers may come from any part of society. In trying to develop an eHealth application to support them we must recognise different carers may require different support and respond to support differently.

One such difference is personality. Personality describes who we are and how we react in different circumstances. There are many ways to measure personality. One of the most popular and reliably validated is the Five-Factor Model (FFM) (Goldberg 1993), which describes an individual's personality as a set of scores on five different factors or traits: Extraversion (I), Agreeableness (II), Conscientiousness (III), Emotional Stability (or Neuroticism) (IV) and Openness to Experience (V). We hypothesize that carers with different 
personalities may require different types and amounts of Emotional Support.

Highly emotionally stable individuals are calm, nonneurotic and imperturbable (John and Srivastava 1999), while neurotic individuals (those with low Emotional Stability) are more likely to worry, feel negative affective states and experience depressive symptoms (Watson 2000, Larsen and Ketelaar 1991, Lahey 2009), and as such may require more support to deal with these emotions. We hypothesize that these individuals will value emotional support more highly as they are more affected by stress.

Astrid et al. (2010) investigated the impact of personality factors on experience with VAs. They found that big five factors Extraversion and Agreeableness had an impact on the users' experience with the VA. They found that people with high agreeableness felt better after interaction with the VA. Extraverted individuals used more words to interact with the VA, implying improved engagement. We hypothesize that individuals with higher agreeableness will rate the VA more favourably than people with low agreeableness.

In this study we aim to explore if emotional support from a computer is more or less supportive than from humans. Yet it is likely that people rate support from a stranger differently than support from a friend. Dunbar's (Dunbar 1998, Hill and Dunbar 2003) studies of personal social networks tell us that an individual (ego) has several bands of friendships each with distinctive qualities that are of a constant size across cultures. Several factors have been identified as influencing the size of an individual's personal network - e.g. income and marriage. It has also been found that without network maintenance regular interaction between members - a friendship will degrade, though kin relationships require less maintenance. Thus, if an individual is not able to invest time in network maintenance then it is likely that they will lose friends (Roberts and Dunbar 2011). We can use the network bands (described by Zhou et al. (2005)) to explore how network closeness affects an emotional support message supportiveness compared to support from a VA.

Previous research has explored how emotional support expectations vary between different members of a social network; what you expect from a close family member is different from a work colleague. Moncur et al (Moncur et al. 2014) demonstrated this in a study where parents of infants in neo-natal intensive care chose to share information differently between people of different emotional proximity. Thus we expect that people will find an emotional support message more or less suitable depending on social network proximity. We anticipate that the Al chatbot will be rated lowest, but may be comparable to support from an acquaintance.
In previous studies, researchers have investigated what types of emotional support are suitable for carers experiencing different stressors (e.g. isolation, physical demand, interruption) (Smith et al. 2014) and first responders experiencing stress (Kindness et al. 2017). They have also investigated how to add emotional context to emotional support messages through gift emoticons (Smith 2015), and how emotional support messages should be tailored to users with high or low emotional stability (Smith et al. 2015). In this study we wanted to explore whether people rated the support message differently if they thought it was coming from a real person or a computer. We also wanted to see if people with different personalities would rate the support differently.

\section{METHODS}

In this study we examine whether social network distance and family/friend relationship have an impact on emotional support supportivenss. We contrast this with computer-provided support. We also investigate whether people's personality has an impact on their ratings.

Ethical approval for the study was obtained from the department ethics committee. Informed consent was obtained from participants prior to participation.

\subsection{Design}

We used a between-subject design. Participants saw one scenario and one message (see Figure 1). The message was depicted as coming from either a computer, a sister or a friend. The sister/friend was either a 'best friend' (close), close acquaintance (medium) or distant acquaintance (distant), as shown in Table 1.

Participants rated their empathy with the scenario (here called 'Sympathy' to disambiguate it from the message category 'Empathy'), to allow us to control for low empathy, as used in (Smith et al. 2014). They rated the support message on 4 Rating Types: scales of helpfulness, appropriateness, sensitivity and effectiveness (all Likert scales from 1 to 9 , see Figure 1, taken from Jones \& Burleson 1997) an They also completed a personality slider task (from sec:sliders) for the Big 5 personality traits. The Independent variables were support provider identity (7 levels: computer, sister-close, friend-close, sister-medium, friend-medium, sisterdistant and friend-distant) and participant personality (a score ranging between 18 and 162 for each personality trait of Openness to Experience, Conscientiousness, Extraversion, Agreeableness and Emotional Stability); the dependent variables were Sympathy (1-7) and Message rating. Qualitative data was also obtained. 


\section{Voluntary Research Study}

\section{Section 2 of 3}

Read and follow the instructions below. Take your time - there are no right or wrong answers; we are interested in what you think.

The following scenario is about Alice, a home carer. Alice is Fred's carer. A home carer is a person who provides regular mental or physical support for someone without formal payment

Today Fred was confused and very upset and Alice comforted him.

Imagine you are Alice.

How well do you think you can empathize with the stress he is experiencing in this situation?

very poorly='l don't understand this situation/would not find this stressful'

Very well='l have experienced a similar situation and understand exactly how stressful it is

Please select

After this occurred, Alice received a message from Sally. Sally is Alice's sister, someone whom Alice gets on well with and usually gets in touch with once a year.

This is the message:

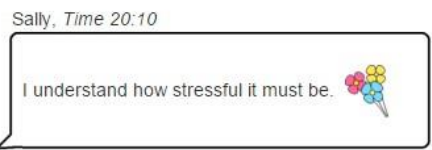

Please rate the support that Sally has given to Alice and explain why you have given this rating.

$\begin{array}{llllllllllll} & & \begin{array}{llllll}1 \\ \text { Appropriateness }\end{array} & \mathbf{3} & \mathbf{4} & \mathbf{5} & \mathbf{6} & \mathbf{7} & \mathbf{8} & \mathbf{9} \\ \text { Helpfulness } & \text { Very Inappropriate } & 0 & 0 & 0 & 0 & 0 & 0 & 0 & 0 & 0 & \text { Very Appropriate } \\ \text { Effectiveness } & \text { Very Ineffective } & 0 & 0 & 0 & 0 & 0 & 0 & 0 & 0 & 0 & \text { Very Effective } \\ \text { Sensitivity } & \text { Very Insensitive } & 0 & 0 & 0 & 0 & 0 & 0 & 0 & 0 & 0 & \text { Very Sensitive }\end{array}$

Please explain why you have given these ratings

Figure 1: Screenshot of Study 2 showing support interaction

\section{Voluntary Research Study}

\section{Section 3: Which person are you most like?}

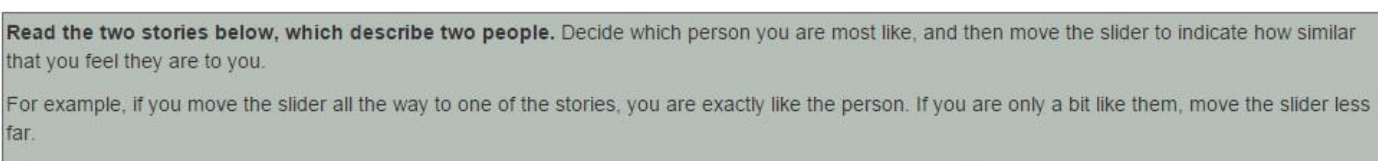

James has a sharp tongue and cuts

others to pieces. He suspects hidden

motives in people. He holds grudges and

gets back at others. He insults and

contradicts people, believing he is better

than them. He makes demands on others.

and is out for his own personal gain.

James tends to be calm and quite likes

exploring new ideas.
Charlie has a good word for everyone,

believing that they have good intentions.

He respects others and accepts people as

they are. He makes people feel at ease.

He is concerned about others, and trusts

what they say. He sympathizes with others

feelings, and treats everyone equally. He is easy to satisfy. Charlie tends to be quite anxious.

Figure 2: Screenshot of the personality measurement for Agreeableness that participants completed in Study 2 
Table 1: The seven conditions describing who the support provider 'Sally' is, and her relationship to Alice (the carer). Relationship description is based on (Zhou et al. 2005)

\begin{tabular}{|l|c|c|}
\hline \multicolumn{1}{|c|}{$\begin{array}{c}\text { Relationship description. "After this occurred, Alice } \\
\text { received a message from Sally. Sally is..." }\end{array}$} & Friend type & Friendship level \\
\hline $\begin{array}{l}\text { An Artificial-Intelligence computer application } \\
\text { (chatbot). }\end{array}$ & computer & computer \\
\hline $\begin{array}{l}\text { Alice's best friend, someone whom Alice depends on to offer } \\
\text { support and advice in a crisis. }\end{array}$ & sister & close \\
\hline $\begin{array}{l}\text { Alice's sister, someone whom Alice depends on to offer } \\
\text { support and advice in a crisis. }\end{array}$ & friend \\
\hline $\begin{array}{l}\text { Alice's friend, someone whom Alice speaks to a few times a } \\
\text { month. }\end{array}$ & sister \\
\hline $\begin{array}{l}\text { Alice's sister, someone whom Alice speaks to a few times a } \\
\text { month. }\end{array}$ & friend \\
\hline $\begin{array}{l}\text { Alice's acquaintance, someone whom Alice gets on well with } \\
\text { and usually gets in touch with once a year. }\end{array}$ & distant \\
\hline $\begin{array}{l}\text { Alice's sister, someone whom Alice gets on well with and } \\
\text { usually gets in touch with once a year. }\end{array}$ & & distant \\
\hline
\end{tabular}

\subsection{Materials}

- One stressful scenario depicting emotional demand and one support message depicting empathy were used (as empathy is considered to be high quality emotional support; Burleson \& Kunkel 1996). These were taken from (Smith et al. 2014) and are also used in (Smith 2015); see Figure 1.

- We used Zhou et al. (2005) to guide us in definitions of relationship closeness (see Table 1), which adhere to Dunbar (1998)'s Social Network Theory.

- We decided to depict the carer's friend/family member as female, as the carer in the scenario is female. This was to avoid a possible confound of participants' interpretations of a Male-Female relationship.

- We described the family member as a sister in order for them to be judged as a peer rather than more senior or junior in the family hierarchy.

- Sliders to measure the big 5 personality traits were taken from (Smith et al. nd) (see Figure 2)

\subsection{Participants}

Participants were recruited from Mechanical Turk (MT nd) and were paid $\$ 0.50$. This was chosen as a good participant pool of people with experience of caring compared to opportunistic sampling, and as it suited our indirect approach. Smith et al. (2014) has previously found that MT provides a rich source of emotional support messages for carers. Participants had to complete an English comprehension test, have an acceptance rate of at least $90 \%$ and reside in the US. There were 140 participants; 2 of these were excluded for incomplete results. This left 79 females, 57 males and 2 undisclosed. 22 were aged 18-25, 77 were 26-40, 36 were 41-65, 2 were over 65 and 1 did not disclose their age. 80 participants had knowledge of care settings: there were 34 informal carers, 15 professional carers and 31 friends of carers in the sample.

\subsection{Procedure}

The procedure is depicted in Figure 1. Participants were first presented with the information sheet consent and consent form. They then provided basic demographics and were screened using the English Comprehension task. They were then randomised to one of the seven conditions (see Table 1).

Participants were told what a carer was and that they would be shown one scenario involving a carer called Alice. They were presented with the scenario and asked to rate their empathy (here called 'Sympathy') with the carer's situation. Then they were introduced to Sally as either Alice's friend, sister or computer (see Table 1) and were asked to rate a short support message that Sally had sent to Alice and explain why they had given those ratings. They were then presented with the Big 5 Personality trait story pairs with a slider to indicate how close they thought they were to one of the people described in the stories (see Figure 2).

This indirect method was chosen as it is difficult to explore stressful situations whilst they occur (both for ethical and practical reasons). We measured 
empathy as a covariate to mitigate that some participants don't empathise with stressful situations; this has been found to be effective in previous studies (Smith et al. 2014).

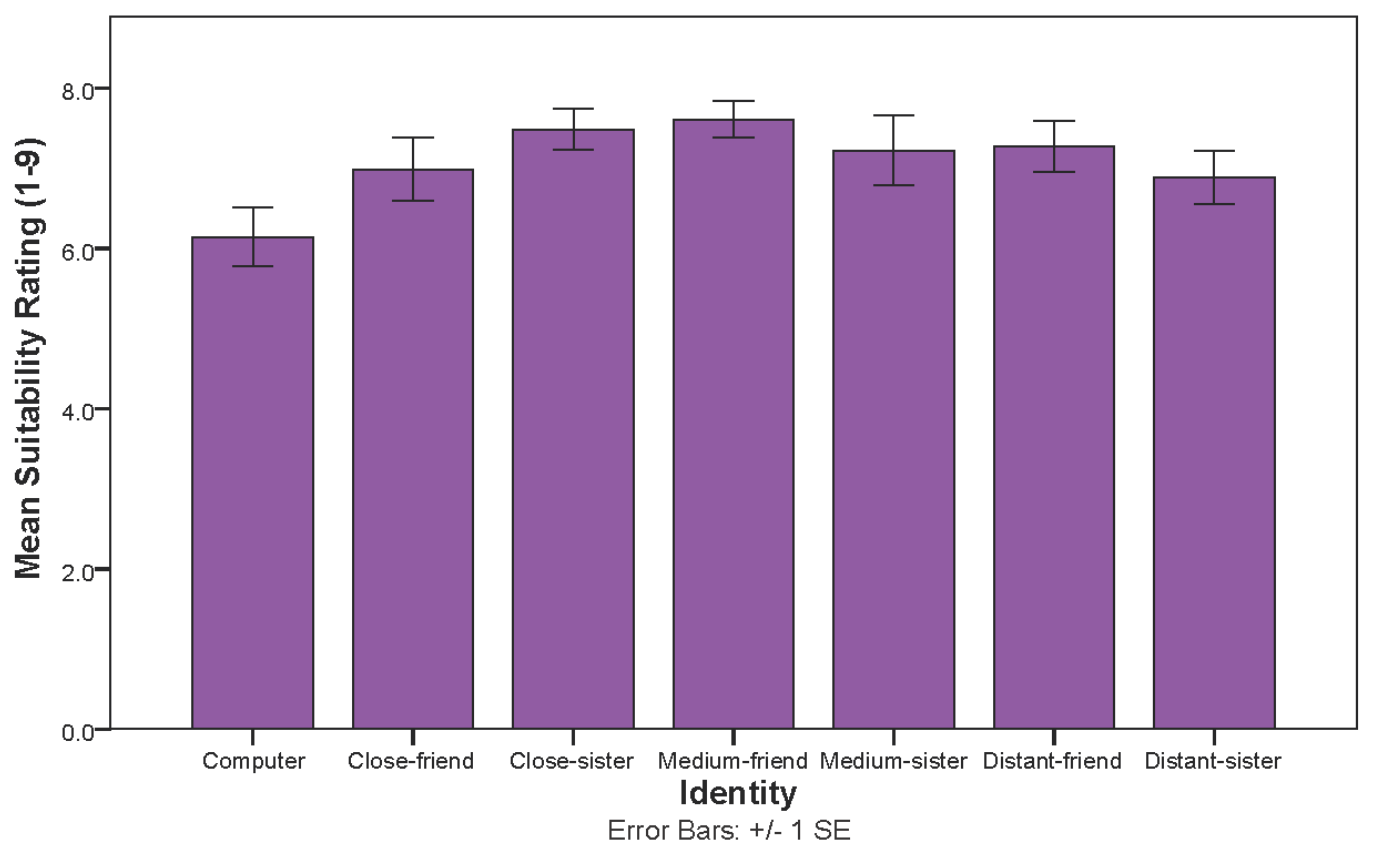

Figure 3: Mean Supportiveness rating for each condition, varying Identity (Computer/Family/Friend) and Relationship Closeness (Close/Medium/Distant).

Table 2: Homogeneous subsets for Identity

\begin{tabular}{|l|l|l|}
\hline \multicolumn{2}{|c|}{ Subset } & Mean \\
\hline 1 & Computer & 6.12 \\
\cline { 2 - 3 } & Distant-sister & 6.88 \\
\cline { 2 - 3 } & Close-friend & 7.02 \\
\hline 2 & Distant-sister & 6.88 \\
\cline { 2 - 3 } & Close-friend & 7.02 \\
\cline { 2 - 3 } & Medium-sister & 7.23 \\
\cline { 2 - 3 } & Distant-friend & 7.28 \\
\cline { 2 - 3 } & Close-sister & 7.45 \\
\cline { 2 - 3 } & Medium-friend & 7.58 \\
\hline
\end{tabular}

\subsection{Hypotheses}

We had the following hypotheses:

- H1 Computer-provided support will be rated lower than human-provided support

- H2 Family support will be rated differently from friend support

- H3 Support from close friends/family will be rated differently than support from distant friends/family

- H4 People with lower emotional stability will rate the support more highly than high emotional stability people

- H5 People with higher agreeableness will rate the computer-provided message more highly than people with low agreeableness.

\section{RESULTS}

An initial review of the data revealed that it was normally distributed and thus suitable for using parametric tests.

\subsection{Effects of Identity and Rating Type on Rating}

A $7 \times 4$ ANCOVA of Identity $\times$ Rating Type (Supportiveness subscales of Effectiveness, Helpfulness, Appropriateness and Sensitivity) was performed on rating, controlled for Sympathy. This was significant for Rating Type $F(3,523)=14.33$, $p<0.05$ and Identity $F(6,523)=5.01, p<0.05$, but not the interaction. The main effects can be seen in Figure 3. Post-hoc tests reveal two homogeneous subsets (see Table 2). Medium-friend, distantfriend, medium-sister and close-sister were significantly higher rated for the same message than the computer. The message was rated as significantly more sensitive and appropriate than effective or helpful.

From this, we find support for $\mathrm{H} 1$ : computergenerated support was rated lower than humanprovided support. We also found some support for $\mathrm{H} 2$ and $\mathrm{H} 3$, that family support was rated differently than friend support, and close support was rated differently than distant - the message from the distant-sister was rated low, while the message from the close-friend was rated low. This shows that 
participants' ratings depended on both the identity of the supporter and the closeness of the relationship.

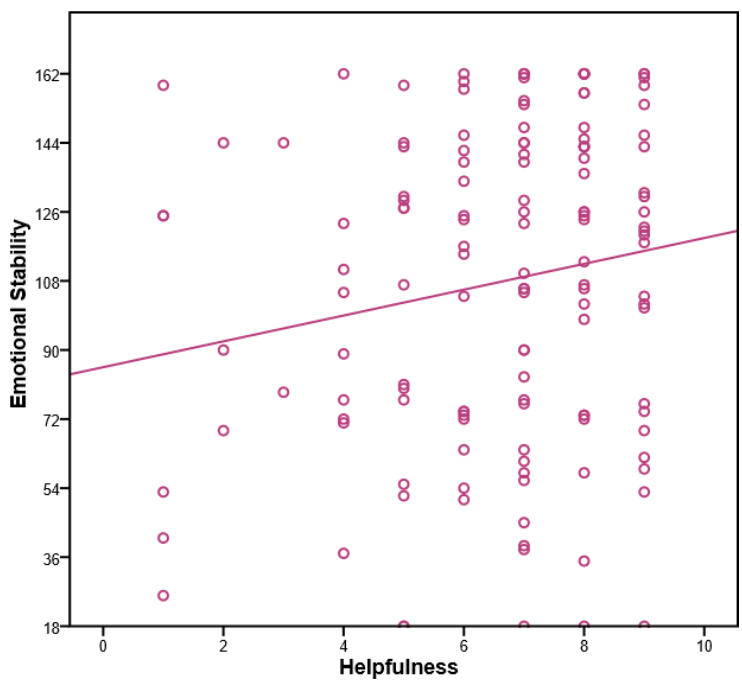

Figure 4: Correlation of Emotional Stability and Helpfulness

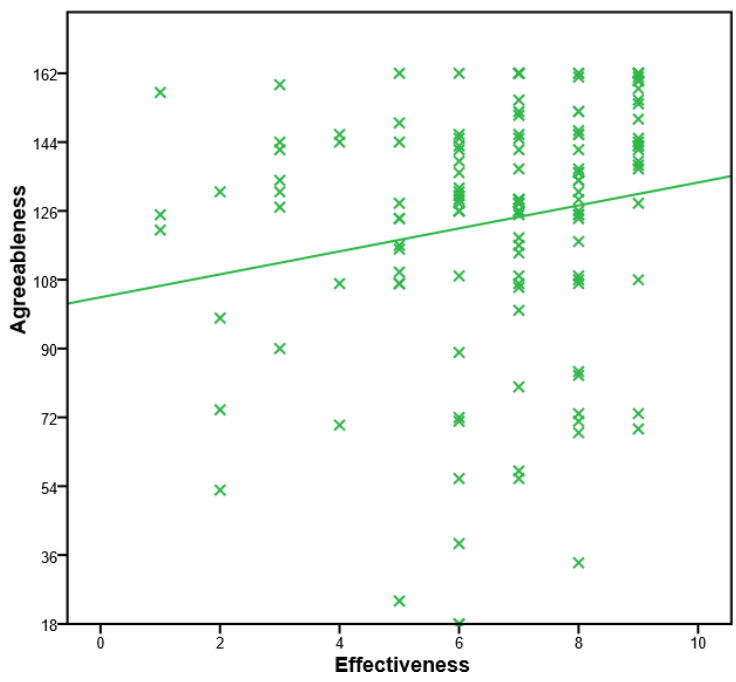

Figure 5: Correlation of Agreeableness and Effectiveness

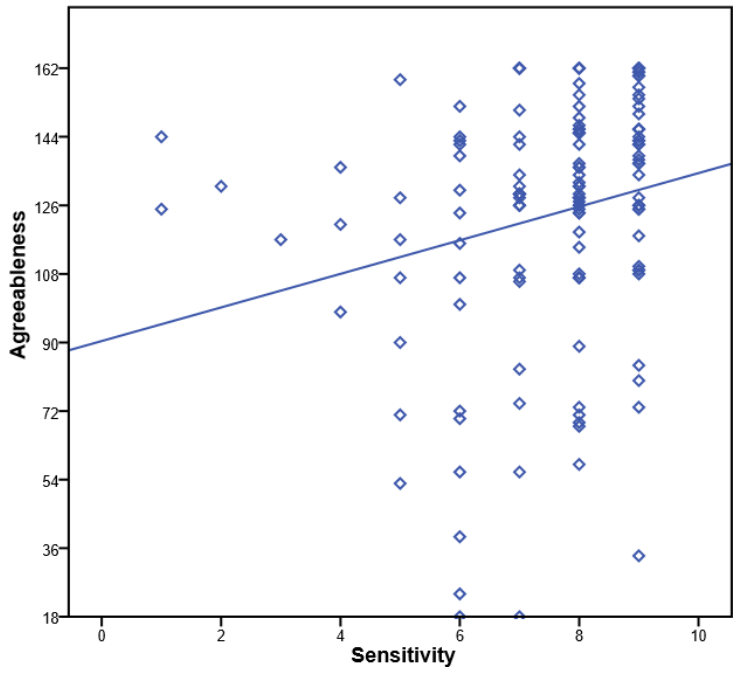

Figure 6: Correlation of Agreeableness and Sensitivity

\subsection{Effects of Personality on Rating}

A Correlation analysis was run between the 5 big five personality trait scores and the 4 rating types, controlling for sympathy through use of a partial correlation analysis. Agreeableness had a positive correlation with Effectiveness $(r(135)=0.17, p=0.05$; see Figure 5) and Sensitivity $(r(135)=0.18, p<0.05$; see Figure 6) and Emotional Stability had a positive correlation with Helpfulness $(r(135)=0.17, p=0.05$; see Figure 4). Additionally, several of the traits correlated with each other (see Table 3). These correlations are small, and seem in line with other observations that the Big Five factors are not completely orthogonal (DeYoung 2006, Saucier 2002, Anusic et al. 2009, Dennis et al. 2012).

\subsection{Effects of Personality, Rating Type and Identity on Rating}

To explore whether the correlations we found for Agreeableness and Emotional Stability varied with Identity, we ran an ANCOVA examining the interaction between Personality, Identity and Rating Type. We found a significant interaction between Rating Type and Emotional Stability $(F(3,366)=3.47$, $\mathrm{p}<0.05)$, but no other effects. This is most likely due to the small sample size and number of statistical tests that have been corrected for.

Thus we found no support for $\mathrm{H} 4$, that people with lower emotional stability will rate the support more highly. Instead we found an indication that people with high emotional stability rated the message slightly higher on helpfulness than people with low emotional stability (see Figure 4). This did not interact with Identity. We also found that people with high agreeableness rated the message higher on effectiveness (see Figure 5) and sensitivity (see Figure 6) than participants with low agreeableness. This provides partial support for $\mathrm{H} 5$, but that this is not specific to VAs - people with high agreeableness are more favourable to an interaction regardless of the identity of the support provider.

\subsection{Thematic Analysis of Comments}

Following our quantitative analysis, we performed a qualitative analysis (using an open-coded thematic analysis) of rating explanations to explore why people had rated the message in certain ways. Our analysis was based on a (compulsory) comment box on the page (see Figure 1); people did not leave extensive comments (no comment was longer than 3 sentences). There were 19-20 participants per condition, so for each condition there is a relatively small pool of comments to analyse. Additionally, many of the participants commented on the general content of the message rather than the relationship between the sender and receiver - we were not interested in these general comments in this study. We found the following themes: 
Table 3: Partial correlations between each Big 5 trait and each rating type of Supportiveness, controlling for Sympathy. Significant correlations are indicated in bold.

\begin{tabular}{|c|c|c|c|c|c|c|c|c|c|c|}
\hline & & Agr & Ext & Conc & OE & ES & Appropriate & Helpful & Effective & Sensitive \\
\hline Agr & $\begin{array}{l}r \\
p\end{array}$ & 1.000 & $\begin{array}{l}0.147 \\
0.086 \\
\end{array}$ & $\begin{array}{l}0.214 \\
0.012 \\
\end{array}$ & $\begin{array}{l}0.216 \\
0.011 \\
\end{array}$ & $\begin{array}{l}0.107 \\
0.213 \\
\end{array}$ & $\begin{array}{l}0.107 \\
0.215 \\
\end{array}$ & $\begin{array}{l}0.134 \\
0.117 \\
\end{array}$ & $\begin{array}{l}0.167 \\
0.050 \\
\end{array}$ & $\begin{array}{l}0.182 \\
0.033 \\
\end{array}$ \\
\hline Ext & $\begin{array}{l}r \\
p\end{array}$ & $\begin{array}{l}0.147 \\
0.086 \\
\end{array}$ & 1.000 & $\begin{array}{l}0.073 \\
0.396 \\
\end{array}$ & $\begin{array}{l}0.126 \\
0.142 \\
\end{array}$ & $\begin{array}{l}0.427 \\
0.000 \\
\end{array}$ & $\begin{array}{l}0.023 \\
0.785 \\
\end{array}$ & $\begin{array}{l}0.140 \\
0.102 \\
\end{array}$ & $\begin{array}{l}0.075 \\
0.386 \\
\end{array}$ & $\begin{array}{l}-0.018 \\
0.836 \\
\end{array}$ \\
\hline Conc & $\begin{array}{l}r \\
p \\
\end{array}$ & $\begin{array}{l}0.214 \\
0.012 \\
\end{array}$ & $\begin{array}{l}0.073 \\
0.396 \\
\end{array}$ & 1.000 & $\begin{array}{r}-0.024 \\
0.781 \\
\end{array}$ & $\begin{array}{l}0.335 \\
0.000 \\
\end{array}$ & $\begin{array}{l}0.024 \\
0.778 \\
\end{array}$ & $\begin{array}{l}0.074 \\
0.388 \\
\end{array}$ & $\begin{array}{l}0.036 \\
0.679 \\
\end{array}$ & $\begin{array}{l}0.047 \\
0.584 \\
\end{array}$ \\
\hline$O E$ & $\begin{array}{l}r \\
p\end{array}$ & $\begin{array}{l}0.216 \\
0.011 \\
\end{array}$ & $\begin{array}{l}0.126 \\
0.142 \\
\end{array}$ & $\begin{array}{r}-0.024 \\
0.781 \\
\end{array}$ & 1.000 & $\begin{array}{l}0.090 \\
0.297 \\
\end{array}$ & $\begin{array}{l}-0.023 \\
0.788 \\
\end{array}$ & $\begin{array}{l}0.047 \\
0.584 \\
\end{array}$ & $\begin{array}{l}0.002 \\
0.978 \\
\end{array}$ & $\begin{array}{l}0.050 \\
0.559 \\
\end{array}$ \\
\hline$E S$ & $\begin{array}{l}r \\
p\end{array}$ & $\begin{array}{l}0.107 \\
0.213 \\
\end{array}$ & $\begin{array}{l}0.427 \\
0.000 \\
\end{array}$ & $\begin{array}{l}0.335 \\
0.000 \\
\end{array}$ & $\begin{array}{l}0.090 \\
0.297\end{array}$ & 1.000 & $\begin{array}{l}0.052 \\
0.548 \\
\end{array}$ & $\begin{array}{l}0.168 \\
0.050\end{array}$ & $\begin{array}{l}0.110 \\
0.202 \\
\end{array}$ & $\begin{array}{c}-0.017 \\
0.843 \\
\end{array}$ \\
\hline Appropriate & $\begin{array}{l}r \\
p\end{array}$ & $\begin{array}{l}0.107 \\
0.215 \\
\end{array}$ & $\begin{array}{l}0.023 \\
0.785 \\
\end{array}$ & $\begin{array}{l}0.024 \\
0.778 \\
\end{array}$ & $\begin{array}{r}-0.023 \\
0.788\end{array}$ & $\begin{array}{l}0.052 \\
0.548 \\
\end{array}$ & 1.000 & $\begin{array}{l}0.449 \\
0.000\end{array}$ & $\begin{array}{l}0.506 \\
0.000 \\
\end{array}$ & $\begin{array}{l}0.658 \\
0.000 \\
\end{array}$ \\
\hline Helpful & $\begin{array}{l}r \\
p\end{array}$ & $\begin{array}{l}0.134 \\
0.117 \\
\end{array}$ & $\begin{array}{l}0.140 \\
0.102 \\
\end{array}$ & $\begin{array}{l}0.074 \\
0.388 \\
\end{array}$ & $\begin{array}{l}0.047 \\
0.584\end{array}$ & $\begin{array}{l}0.168 \\
0.050\end{array}$ & $\begin{array}{l}0.449 \\
0.000\end{array}$ & 1.000 & $\begin{array}{l}0.862 \\
0.000 \\
\end{array}$ & $\begin{array}{l}0.592 \\
0.000 \\
\end{array}$ \\
\hline Effective & $\begin{array}{l}r \\
p \\
\end{array}$ & $\begin{array}{l}0.167 \\
0.050 \\
\end{array}$ & $\begin{array}{l}0.075 \\
0.386 \\
\end{array}$ & $\begin{array}{l}0.036 \\
0.679 \\
\end{array}$ & $\begin{array}{l}0.002 \\
0.978 \\
\end{array}$ & $\begin{array}{l}0.110 \\
0.202 \\
\end{array}$ & $\begin{array}{l}0.506 \\
0.000 \\
\end{array}$ & $\begin{array}{l}0.862 \\
0.000 \\
\end{array}$ & 1.000 & $\begin{array}{l}0.646 \\
0.000 \\
\end{array}$ \\
\hline Sensitive & $\begin{array}{l}r \\
p\end{array}$ & $\begin{array}{l}0.182 \\
0.033\end{array}$ & $\begin{array}{r}-0.018 \\
0.836\end{array}$ & $\begin{array}{l}0.047 \\
0.584\end{array}$ & $\begin{array}{l}0.050 \\
0.559\end{array}$ & $\begin{array}{r}-0.017 \\
0.843\end{array}$ & $\begin{array}{l}0.658 \\
0.000\end{array}$ & $\begin{array}{l}0.592 \\
0.000\end{array}$ & $\begin{array}{l}0.646 \\
0.000\end{array}$ & 1.000 \\
\hline
\end{tabular}

\subsubsection{Al diminishes support.}

When informed that 'Sally' was 'an ArtificialIntelligence computer application (chatbot)', participants had divided opinions as to whether the support was suitable or not. Five participants thought that a computer application could be helpful: 'even though it is just Al it could still be helpful', though the support would be 'diminished somewhat because Sally is a chatbot'. Four participants claimed that Sally 'cannot possibly understand how anyone feels', thus the message's impact would be reduced. One participant went as far as saying the would find this message from a computer 'weird and creepy.'

\subsubsection{Offering to help would be appreciated.}

The quantitative analysis showed that participants overall felt that Sally was less helpful and effective than appropriate and sensitive in her message. This is born out in the comments. In the close and medium conditions, participants suggested that Sally 'could have been more helpful by offering to help.' One participant goes as far as suggesting that 'as a sister, Sally could have provided more input and offered her assistance'; it is a family member's duty to help. There is no expectation that distant friends or family should offer practical assistance.

\subsubsection{Acknowledging feelings is helpful.}

Nine participants felt that the support message acknowledged and validated Alice's stress. This, in itself, is helpful: 'While Sally isn't doing anything concrete to help Alice, just the fact that she's acknowledging it's a tough job and being supportive is very helpful.'

\subsubsection{Support from distant friends.}

Four participants commented that the distant sister/friend's message would not be very supportive because 'how can someone be supportive if they're in your life that little?'. Contrastingly, two participants thought it was nice of someone they were not close with to offer support: 'it's good for someone that I don't speak to often to actually acknowledge the situation.'

\subsubsection{Brevity and Personalisation.}

Nine participants commented that the message was short or impersonal. Some participants were critical of this: 'seems like a blanket statement that adds nothing to show that she really does understand how she feels,' while others thought it was nevertheless effective: 'It's a very simple message, but it shows a lot in letting someone know that they're not alone.'

\subsubsection{Familial expectations.}

Two participants highlighted that Alice's sister ought to provide better support because she was her sister: 'I think being her sister she could have expressed sympathy in a better way.' Another participant liked the message and stated, 'That is what family should do'. This indicates that for some participants, family members have an obligation to be supportive. 


\section{DISCUSSION AND CONCLUSION}

In this study we found that the identity of the support giver has an impact on the ratings of supportiveness. People have different expectations of different members of their social network - family members are expected to provide support regardless of closeness, and close friends should provide good quality support. A thematic analysis of comments revealed that people prefer emotional support from a human; they like empathy; support from close friends means more; they prefer personalised support; and they have higher expectations from family over friends. This is to be expected - it is well established that there is an expectation/obligation of help and support from family (e.g Parrott and Bengtson 1999).

We found that people rated the emotional support worse if it came from a computer; however, it was still rated as good support by most participants, with a mean rating of 6.12 (SD 1.61) on a 1 to 9 likert scale of supportiveness (see Figure 3 ). This is an improvement on the mean rating of 6.00 that was found by Smith et al. (2014), where they only presented users with the scenario without any information about the sender of the support message. This implies that knowing that a message comes from a computer does not diminish support. While it is to be expected that people tend to treat VAs like real people (Reeves and Nass 1996), this study provides evidence that emotional support messages from virtual agents are also subject to this personification effect.

We found three weak effects for personality which did not vary with Identity. This supports the results in (Smith 2016), that personality does not have a big impact on emotional support. We found a correlation between Emotional Stability and helpfulness, implying that people with higher emotional stability find emotional support more helpful, and people with high agreeableness are generally more favourable to text-based emotional support than people with lower agreeableness. This provides further support for (Astrid et al. 2010), that agreeableness affects human-computer interactions.

The work presented has several limitations. Firstly, the study only considered one type of stressful scenario, namely Emotional Demand. Validated scenarios for other stressors have been produced (Smith et al. 2014), and these could be used to investigate the impact of stressor. Secondly, the study only used one particular support message. Researchers have validated many support message types and instances for different types of stressors, which can be used in follow-on studies (Smith et al. 2014). Thirdly, the study only investigated the Big Five personality traits. Follow-on studies could consider other personality traits such as self-esteem and resilience. Fourthly, we only considered one instance of emotional support. Follow-on studies could look at sequences of support messages for when people experience multiple stressful situations over time. Fifthly, only textual support messages were used. Follow-on studies could investigate the impact of adding different emoticons (e.g. those proposed in (Smith 2016)), a visual representation of the agent, or emotional expressions by the agent. Finally, the study was indirect: asking participants' opinions on support messages for an informal carer in a particular stressful situation. A follow-on study could repeat this using participants who were actually experiencing the stressful situation themselves.

We did not explore the impact of personality in our thematic analysis. While this would be interesting, it would require a far larger sample; it is difficult to isolate which traits cause which opinions.

In this work we only compared a computer to known others. It would be interesting to see how Al support compared to an unknown other (e.g. volunteer from charity, healthcare worker); this might be more comparable to computer-generated support.

The findings of our study lead us to believe that a computer can provide acceptable emotional support for people in stressful situations. The crowd-sourcing methodology we used was particularly useful in investigating this without having to go through the long process of face-to-face co-design with carers (we would have needed very many carers if we wanted to investigate personality adaptation). Therefore we can now go on to implement and test a system with real informal carers.

The implications of this work for emotional support agents are encouraging - an emotional support message delivered by a VA is appreciated by users, and is rated at least as supportive as a message from an acquaintance. However, there are individual differences: some users do not like support from a computer at all. Further investigations should explore what individual differences impact upon this, so that the use of a VA to deliver emotional support can be appropriately tailored to the user.

\section{REFERENCES}

Anusic, I., Schimmack, U., Pinkus, R. T., and Lockwood, P. (2009). The nature and structure of correlations among big five ratings: The haloalpha-beta model. Journal of Personality and Social Psychology, 97(6):1142.

Astrid, M., Kr"amer, N. C., and Gratch, J. (2010). How our personality shapes our interactions with virtual characters-implications for research and development. In International Conference on Intelligent Virtual Agents, pages 208-221. Springer. 
Burleson, B. R. and Kunkel, A. W. (1996). The socialization of emotional support skills in childhood. In Handbook of social support and the family, pages 105-140. Springer.

Caqueo-Urízar, A., Gutíírrez-Maldonado, J., and Miranda-Castillo, C. (2009). Quality of life in caregivers of patients with schizophrenia: a literature review. Health and Quality of Life Outcomes, 7(1):1.

Carers UK (2015). Facts about carers.

Dennis, M., Masthoff, J., and Mellish, C. (2012). The quest for validated personality trait stories. In IUI, pages 273-276. ACM.

DeYoung, C. G. (2006). Higher-order factors of the big five in a multi-informant sample. Journal of personality and social psychology, 91(6):1138.

Dunbar, R. (1998). The social brain hypothesis. brain, 9(10):178-190.

Goldberg, L. (1993). The structure of phenotypic personality traits. American Psychologist, 48:2634.

Hill, R. A. and Dunbar, R. I. (2003). Social network size in humans. Human nature, 14(1):53-72.

Jones, S. M., \& Burleson, B. R. (1997). The impact of situational variables on helpers' perceptions of comforting messages: An attributional analysis. Communication Research, 24(5), 530-555.

John, O. P. and Srivastava, S. (1999). The big five trait taxonomy: History, measurement, and theoretical perspectives. Handbook of personality: Theory and research, 2(1999):102138.

Kindness, P., Masthoff, J., and Mellish, C. (2017). Designing emotional support messages tailored to stressors. International Journal of HumanComputer Studies, 97:1-22.

Lahey, B. B. (2009). Public health significance of neuroticism. American Psychologist, 64(4):241.

Larsen, R. J. and Ketelaar, T. (1991). Personality and susceptibility to positive and negative emotional states. Journal of personality and social psychology, 61(1):132.

Meyer, D. K. and Turner, J. C. (2002). Discovering emotion in classroom motivation research. Educational psychologist, 37(2):107-114.

Moncur, W., Masthoff, J., Reiter, E., Freer, Y., and Nguyen, H. (2014). Providing adaptive health updates across the personal social network. Human- Computer Interaction, 29(3):256-309.

MT (n.d.). Amazon mechanical turk.

Parrott, T. M. and Bengtson, V. L. (1999). The effects of earlier intergenerational affection, normative expectations, and family conflict on contemporary exchanges of help and support. Research on Aging, 21(1):73-105.

Reeves, B. and Nass, C. I. (1996). The media equation: How people treat computers, television, and new media like real people and places. Cambridge university press.

Roberts, S. G. and Dunbar, R. I. (2011). The costs of family and friends: an 18month longitudinal study of relationship maintenance and decay. Evolution and Human Behavior, 32(3):186-197.

Saucier, G. (2002). Orthogonal markers for orthogonal factors: The case of the big five. Journal of Research in Personality, 36(1):1-31.

Savage, S. and Bailey, S. (2004). The impact of caring on caregivers' mental health: a review of the literature. Australian Health Review, 27(1):111.

Schoenmakers, B., Buntinx, F., and Delepeleire, J. (2010). Factors determining the impact of caregiving on caregivers of elderly patients with dementia. a systematic literature review. Maturitas, 66(2):191-200.

Scholten, M. R., Kelders, S. M., and van GemertPijnen, J. E. (2017). A scoped review of the potential for supportive virtual coaches as adjuncts to selfguided web-based interventions. In International Conference on Persuasive Technology, pages 43-54. Springer.

Smith, K. A. (2015). Assessing the supportiveness of gift emoticons in care scenarios. In Extended Abstract of $\mathrm{CHI} 2015$, pages 151-156. ACM.

Smith, K. A. (2016). Exploring personalised emotional support. PhD thesis, University of Aberdeen.

Smith, K. A., Dennis, M., Masthoff, J., and Tintarev, N. (n.d.). A method of conveying and measuring personality traits using personality stories. In progress.

Smith, K. A., Masthoff, J., Tintarev, N., and Moncur, W. (2014). The development and evaluation of an emotional support algorithm for carers. In Intelligenza Artificiale, volume 8:2, pages 181196. IOS Press.

Smith, K. A., Masthoff, J., Tintarev, N., and Moncur, W. (2015). Adapting emotional support to personality for carers experiencing stress. In PATH at- UMAP 2015 Adjunct Proceedings. CEUR-WS.

Vitaliano, P. P., Zhang, J., and Scanlan, J. M. (2003). Is caregiving hazardous to one's physical health? a meta-analysis. Psychological Bulletin, 129(6):946-72. Watson, D. (2000). Mood and temperament. Guilford Press. 
World Health Organization and others (2016). Global diffusion of ehealth: making universal health coverage achievable: report of the third global survey on ehealth.

Zhou, W.-X., Sornette, D., Hill, R. A., and Dunbar, R. I. (2005). Discrete hierarchical organization of social group sizes. Proceedings of the Royal Society of London B: Biological Sciences, 272(1561):439-444. 